\title{
Risk Factors for Posttraumatic Stress Disorder in Haitian Students
}

\author{
Gretel Silvestre1, Pascale Anacréon1, Michèle Théodore1, Emmanuel Silvestre1, \\ Eugenia Garcia-Dubus ${ }^{2}$ \\ ${ }^{1}$ Pontificia Universidad Católica Madre y Maestra, Santiago, Dominican Republic \\ ${ }^{2}$ John Jay College of Criminal Justice, New York, USA \\ Email: gsilvestre@pucmm.edu.do
}

Received 12 April 2014; revised 8 May 2014; accepted 2 June 2014

Copyright (C) 2014 by authors and Scientific Research Publishing Inc.

This work is licensed under the Creative Commons Attribution International License (CC BY).

http://creativecommons.org/licenses/by/4.0/

(c) (7) Open Access

\begin{abstract}
The aim of this study was to assess the prevalence of posttraumatic stress disorder (PTSD) in undergraduate students after the Haiti earthquake on January 12, 2010, as well as to identify the risk factors involved in the development of PTSD symptoms in this sample. Evaluations concerning depression, anxiety, risk and protective factors, and PTSD symptoms were conducted in 246 Haitian undergraduate students enrolled in a Dominican private university. Results indicate a prevalence of $36 \%$ for PTSD 2 years after the earthquake, with a high prevalence also of depression (31.7\%) and anxiety (21.1\%). Some of the risk factors identified are being female, a history of psychiatric treatment, and the amount of personal and material losses. The instauration of crisis management and follow-up protocols after traumatic events was deemed to be needed. Further research is necessary to study the long-term effects of this tragedy, not only in undergraduate students, but also in the working class Haitian immigrants living in the Dominican Republic.
\end{abstract}

\section{Keywords}

Epidemiology, Ethiology-Risk and Protective Factors, Earthquake, PTSD, Haiti

\section{Introduction}

On January 12, 2010, at 5:55 pm (local time), an earthquake of 7.3 magnitude in Richter's scale shook Haiti. The epicenter was located a mere 15 kilometers (about 9 miles) away from Port-au-Prince. The quake lasted longer than a minute and a tsunami warning was active for two hours in several Caribbean nations including Cuba, the Dominican Republic and the Bahamas (“A strong earthquake reduces to rubble Haiti’s capital”, 2010). 
At the time of the earthquake, Haiti had the lowest United Nations Human Development Index of the western hemisphere (Brown, Ripp, \& Kazura, 2012).

As a consequence of the earthquake the city of Port-au-Prince was destroyed, resulting in approximately 19 million cubic meters of rubble and debris. More than 220 thousand dead, 500 thousand homeless and more than 2 million people displaced (Brown et al., 2012), all of this in addition of a history marred by political and social violence, and over $77 \%$ of the population living in conditions of extreme poverty (Pan American Health Organization [PAHO] "Health situation analysis and trends summary", 2009).

Posttraumatic Stress Disorder (PTSD) has always aroused a lot of interest, especially after natural disasters. This disorder has been defined as an anxiety disorder with extremely debilitating effects, which usually happens after a major trauma. Such a trauma includes exposure to a violent or dangerous event, such as military combat, natural disasters, terrorist acts, severe accidents, rapes and child abuse. The trauma is experienced with intense fear, helplessness or horror and can be experienced directly or indirectly (American Psychiatric Association [APA], 2000).

There are several types of traumatic events that can lead to PTSD. These are classified into four categories: abuse (mental, sexual, verbal or physical), catastrophes (accidents, natural disasters, and terrorism), violent attack (assault, abuse, domestic violence and rape) and war, battle and combat (death, explosion, shooting). Each of these events can cause the emotional reaction associated with PTSD (APA, 2000).

Tural et al. (2004) explain how the closeness of danger, of being hurt, the level of damage of buildings exposed to the earthquake, being trapped under the rubble, the death of a family member or seeing a dead body are all factors associated with the development of PTSD, while participating in rescue or being alone during the earthquake are not.

The development of PTSD symptoms is related with pre, peri and post traumatic events, while problems related to the recovery linked with events occurred during and after the earthquake are not (Schnurr, Lunney, \& Sengupta, 2004), this is why is essential to assess subjects about events that occurred long ago, like trauma history. Being female and having a minority status are also being identified as risk factors (Brewin, Andrews, \& Valentine, 2000), as are the kind and degree of personal (Chou et al., 2007; Liu et al., 2010; Parvaresh \& Bahramnezhad, 2009) and material loss (Chou et al., 2007; Liu et al., 2010).

There is also a link between developing PTSD and psychological factors such as a history of mental illness (Brewin et al., 2000), anxiety sensibility (McNally, 2008), general negative affect (Zhang \& Ho, 2011) and sleep disturbances (Chou et al., 2007).

Also, as indicated by Armenian et al. (2000), there are some protective factors such as a higher level of education, not being alone during the earthquake and being able to make new friends after the fact.

Low PTSD prevalence rates have been reported among disaster survivors assessed with diagnostic interviews, with the lowest prevalence rates (i.e. $4 \%$ - $8 \%$ ) being found after natural disasters such as floods, volcano eruptions, landslides and tornadoes (McMillen, North, \& Smith, 2000). Some of the reports provided by Tural et al. (2004) suggest that PTSD is more common in developing countries, with a higher risk for severe and very severe deterioration in developing countries as compared to the United States (78\% vs. $25 \%)$. It is thought that developed countries, like Australia and the United States, have a lower prevalence of the disorder because they have the infrastructure for the prevention of devastations and are better organized at pre and post event, with rescue teams and rescue rates superior to those found in developing countries (Salcioglu, Basoglu, \& Livanou, 2007).

Despite the lower PTSD prevalence rates following a natural disaster, we should consider that the characteristics of the population and the country affected may also affect prevalence rates. For this reason, this study was conducted with the aim of examining the prevalence and risk factors associated with the development of PTSD symptoms following the Port-au-Prince earthquake of January 12, 2010 in a sample of Haitian undergraduate students enrolled in the Pontificia Universidad Católica Madre y Maestra (PUCMM), a private university in the Dominican Republic.

Due to the localization and characteristics of the island La Hispaniola, the Dominican Republic and Haiti maintain an unique relationship, with the former becoming the first choice for Haitian youth when in search for a higher education, when fleeing the various socio-political issues or when trying to get a job that represents a better quality of life, all this regardless of the long-term conflict between the two nations over cultural and territorial issues.

Hence, the Dominican Republic was the first to respond after the earthquake, and is still concerned about the post-earthquake living conditions of the Haitian people. This study represents an attempt to gain a better comprehension of what is happening with a considerable percentage of Haitian university students in the Dominican Republic. 


\section{Method}

\subsection{Participants and Procedure}

This study was conducted after the earthquake of January 12, 2010 in Haiti. Specifically, data were collected between August 2011 and March 2012 at PUCMM both in the Santo Domingo and Santiago campuses. The final sample consisted of 246 Haitian students, out of a total of 523 enrolled in the university at the beginning of the study, for $47 \%$ of the population. For a $95 \%$ Confidence Level, this sample produced an acceptable Confidence Interval of $4.59 \%$. Students were recruited through social networking sites and with the help of several leaders within the community. Once recruited, participants were met at a set time and date to complete the survey anonymously after providing informed consent. The sample included $55.7 \%$ male students and $44.3 \%$ female. The mean age was 23.1 years $(s d=3.10)$. Most were medical students $(27.2 \%)$, which is consistent with the Haitian students' enrollment in school. More than $60 \%$ came from Port-au-Prince.

\subsection{Instruments}

- PTSD Symptom Scale (PSS-I) (Cohen, Kamark, \& Mermelstein, 1983). The original instrument is a semistructured interview that assesses the severity of current PTSD symptoms through 17 items which correspond to the 17 symptoms described in the Diagnostic and statistical manual of mental disorders (4th ed., text rev; DSM-IV-TR; APA, 2000). The scale provides a total score as well as sub-scale scores on re-experiencing, avoidance and hyperarousal symptoms (Foa \& Tolin, 2000).

- Beck Depression Inventory (Beck, Ward, Mendelson, Mock, \& Erbaugh, 1961). This is a 21-item self-administered questionnaire that assesses a range of depressive symptoms. Of the 21 items, 15 refer to psychological-cognitive symptoms and the remaining 6 to somatic-vegetative symptoms. It assesses the severity/ intensity of symptoms in a time frame that references the current time and the previous week. The maximum score is 63 points (Beck, 2006).

- Beck Anxiety Inventory (Beck, Epstein, Brown, \& Steer, 1988). This is a screening measure that evaluates the severity of the anxiety. It was designed to reduce the overlap between the scales of depression and anxiety, assessing those symptoms of anxiety that are less shared with depression. It assesses both cognitive and somatic symptoms, distinguishing between anxious and non-anxious patients. The maximum score on the scale is 63 points (Beck \& Steer, 1993).

- Questionnaire Adapted to the Sample and Earthquake. This questionnaire was developed specifically to collect demographic information from the participants. The questionnaire was intended to gather information about demographic variables, characteristics of the subject, and factors pre, peri and post earthquake.

\subsection{Data Analysis}

Data analyses were performed using the software Statistical Package for Social Sciences 17.0 (SPSS 17.0). Following the current trend of the scientific literature, both the effect size and the power of our analysis are reported (Cohen, 1992).

First, in order to create a norm to interpret the scale, cut points for the PSS-I were calculated to establish three PTSD severity levels. Scores that fell under the standard error of the mean (11.292) were deemed to be Low. Those falling within the standard error of the mean (12.59) interval were deemed to be Moderate and those falling above the standard error of the mean (13.887) were designated as High.

The scales measures as the demographics age and college year, and the risk factor mean of relatives lost, were analyzed using a one-way ANOVA to compare the means among the different levels of PTSD (high-moderatelow). The rest of the risk factors studied (sex, background, emotional losses, material losses, etc.) were Nominal measures and were analyzed using crosstabs and Chi-square.

In the data collection instrument, the dependent variables were represented by the symptoms of PTSD, as measured by the PSS-I Scale (Foa \& Tolin, 2000). Demographic variables were used as independent variables.

\section{Results}

\subsection{Prevalence}

The prevalence of PTSD symptoms among the participating students was considered as the percentage which 
presented a High level of PTSD symptoms as measured by the PSS-I. This prevalence reached $36.2 \%$ two years after the earthquake.

Cut points for the BDI and anxiety inventory were determined using the same procedure described above for the PSS-I. The prevalence of high level of anxiety symptoms, as measured by the anxiety inventory, was $21.1 \%$. The prevalence of high levels of depression symptoms, as measured by the BDI was $31.7 \%$, more similar to that of PTSD symptoms.

\subsection{Scale Measures}

Table 1 includes two demographic variables and one risk factor that were collected as scales measures. The table contains the values of the calculated statistics, effect sizes, and power.

Table 1 shows that there were no differences among age means through the PTSD Levels. Nevertheless, when comparing the means in the PSS-I scale across the amount of years spent in college we found that as the year of enrollment advances, there is a statistically significant tendency for PTSD scores to be lower $(p<0.01)$. Nevertheless, the effect size of the college year is small, although the power of the analysis is high.

Students classified in the High level of PTSD reported a significantly higher average number of family members killed in the earthquake (0.67) than the average reported by students at both the Moderate (0.21) and Low (0.25) levels of PTSD. The Tamhane's correction was used for this multiple comparison in order to minimize type I error. According to Table 2, the overall effect size of the PTSD was moderate and the power was very high.

\subsection{Nominal Measures}

Table 2 includes one demographic variable and 12 risk factors that were collected as nominal measures.

A significant difference among the levels of PTSD across genders was found. There are more women (43.1\%) than men (30.7\%) in the High PTSD level and more men (63.5\%) than women (46.8\%) in the Low PTSD level.

Table 1. Scale measures.

\begin{tabular}{ccccccc}
\hline Variable & $F$ & $g l$ & $p<$ & Effect size f & Power \\
\hline Demographics: Age & 0.332 & 2 & $\boldsymbol{n s}$ & & & \\
Demographics: College year & 9.021 & 1 & 0.01 & 0.038 & 0.85 \\
Emotional loss: Means of relatives lost & 8.955 & $2 / 242$ & 0.001 & 0.259 & 0.959 \\
\hline
\end{tabular}

Table 2. Nominal measures.

\begin{tabular}{|c|c|c|c|c|c|}
\hline Variable & $\chi^{2}$ & $d f$ & $p<$ & Effect size w & Power \\
\hline Demographics: Sex & 7.05 & 2 & 0.05 & 0.168 & 0.653 \\
\hline Background: Family mental history & 6.806 & 2 & 0.05 & 0.178 & 0.705 \\
\hline Geographic location at the earthquake: Haiti or abroad & 8.975 & 2 & 0.05 & 0.191 & 0.769 \\
\hline Behavior at the event: Fear level & 32.334 & 8 & 0.001 & 0.367 & 0.994 \\
\hline Behavior at the event: Exposure to injured people & 6.94 & 2 & 0.05 & 0.168 & 0.652 \\
\hline Emotional loss: Loss of a first degree relative & 11.337 & 2 & 0.01 & 0.214 & 0.863 \\
\hline Emotional loss: Loss of friends or neighbors & 6.908 & 2 & 0.05 & 0.166 & 0.639 \\
\hline Material loss: Changing housing situation & 15.316 & 2 & 0.001 & 0.25 & 0.947 \\
\hline Material loss: Loss of family business & 15.316 & 2 & 0.001 & 0.351 & 0.999 \\
\hline Material loss: Level of loss of family business & 28.955 & 6 & 0.001 & 0.353 & 0.993 \\
\hline Material loss: Economic difficulties resulting from the earthquake & 25.529 & 6 & 0.001 & 0.319 & 0.978 \\
\hline $\begin{array}{l}\text { Material loss: Extent to which their monthly income were } \\
\text { affected by the earthquake }\end{array}$ & 16.579 & 4 & 0.01 & 0.273 & 0.925 \\
\hline
\end{tabular}


However, it should be noted that the effect of the sex is small despite the power of the analysis being mediumhigh.

The results on the background measure on family mental health history show that the majority of students with prior family history of mental illness (53.3\%) are classified within the High level of PTSD. Also, the majority of the students without prior family history of mental illness (57.8\%) are classified within the Low level of PTSD. The analysis of the family mental history shows a small effect size and a high power.

We compared the proportions of students who were in Haiti or abroad at the time of the earthquake among the different levels of PSTD. The majority of the students (60.8\%) who were abroad at the time of the earthquake were classified among the Low level of PTSD, while almost a half (47.3\%) of those who were in Haiti belongs to the High level of PTSD. This analysis showed a small effect but a high power.

We asked participants to provide an estimate of the magnitude of the fear experienced during the earthquake on a 5-point Likert scale ranging from nothing to extreme. The great majority of students who estimated their level of fear in nothing (85.7\%) were classified within the Low PTSD level, while the majority of students who estimated their fear as extreme (58.7\%) were in the High PTSD level. This time the effect size of the level of PTSD was medium (0.367) and the power of the analysis very high. However, the effect size of this analysis was small and the power was medium high.

With respect to the risk factor concerning exposure to injured people, we found that the majority of students who were not exposed to injured people (74.4\%) were classified within the Low level of PTSD. It is important to note that regarding the exposure to cadavers or body parts, the majority of those exposed (53.4\%) and those who were not exposed (64.3\%), were in the Low level of PTSD, with no significant difference between them $\left(\chi^{2}(2)=4.375, n s\right)$.

The results concerning the two emotional loss factors included in Table 2 are also consistent with each other. Regarding the loss of a first-degree relative, the results indicated that, while most of those who lost someone (52.1\%) were classified in the High level of PTSD, most of those who did not lose anyone (61.1\%) were in the Low level of PTSD. The effect size of this analysis is medium and its power is high.

Also, regarding the loss of friends or neighbors due to the earthquake, we found that most of those who did not lose friends or neighbors (66\%) were classified in the Low level of PTSD, while at the High level of PTSD up to $40 \%$ lost a friend or neighbor in the earthquake. Nevertheless, the effect size of this analysis was small and the power medium high.

With respect to material losses five types were examined: loss of housing, loss of family business, level of loss of family business, financial difficulties resulting from the earthquake, and effect on monthly income. In all cases a larger material loss was associated with being classified as High on the PTSD scale. Conversely, students who reported their material losses to be on the lower end of the scale were more likely to be classified as Low on the PTSD scale. Percentages are reported on Table 3, while Chi-square, $g l, p$, effect size and power are reported in Table 2.

\section{Discussion}

The prevalence rate of severe PTSD symptoms two years after the earthquake was $36 \%$ among Haitian PUCMM's students. This rate is higher of that of teenagers' students one month after the 2008 Sichuan earthquake, which had an 8.5 magnitude in the Ritcher scale. Likewise, our sample presents a higher prevalence of depression symptoms, $31.7 \%$ vs. $26.6 \%$ in the Sichuan sample (Lau et al., 2010).

However, the prevalence rate is slightly lower in comparison with a preliminary study completed a year after the event (36\% vs. 41\%) (Silvestre, Anacréon, Théodore, \& Silvestre, 2011). This tendency to drop with time is expected and in line with previous findings. For example, in the case of young Chinese students in Sichuan, the drop in prevalence was similar, from $11.2 \%$ at 4 months to $5.7 \%$ one year after the earthquake (Lau et al., 2010). It is possible that various factors affecting this particular group are responsible for the high prevalence of PTSD symptoms found. The effect of being from a developing country (Tural et al., 2004), having minority status in the country were the study was conducted (Brewin, Andrews, \& Valentine, 2000), as well as living far from their families (Parvaresh \& Bahramnezhad, 2009), cannot be denied.

The students in this sample reported mainly symptoms related with avoidance and hyperarousal. In addition, those who reported having more PTSD symptoms also endorsed more anxiety and depression symptoms. This is not surprising since these symptoms usually coexist (McMillen, North, \& Smith, 2000; Pérez Benítez et al., 
Table 3. Distribution of material losses among PTSD levels.

\begin{tabular}{|c|c|c|c|c|}
\hline \multirow[b]{2}{*}{ Variable } & \multirow[b]{2}{*}{ Degree } & \multicolumn{3}{|c|}{ PTSD level } \\
\hline & & Low & Medium & High \\
\hline \multirow{2}{*}{ Change of housing situation } & No & $64.1 \%$ & $9.2 \%$ & $26.8 \%$ \\
\hline & Yes & $42.9 \%$ & $5.5 \%$ & $51.6 \%$ \\
\hline \multirow{2}{*}{ Loss of family business } & No & $63.5 \%$ & $7.5 \%$ & $29.0 \%$ \\
\hline & Yes & $20.5 \%$ & $9.1 \%$ & $70.5 \%$ \\
\hline \multirow{5}{*}{ Level of loss of family business } & None & $64.7 \%$ & $7.8 \%$ & $27.5 \%$ \\
\hline & Slight & $63.6 \%$ & $3.0 \%$ & $33.3 \%$ \\
\hline & Moderate & $26.3 \%$ & $15.8 \%$ & $57.9 \%$ \\
\hline & Severe & $23.3 \%$ & $6.7 \%$ & $70.0 \%$ \\
\hline & None & $74.2 \%$ & $4.5 \%$ & $21.3 \%$ \\
\hline \multirow{3}{*}{ Economic difficulties resulting from the earthquake } & Slight & $49.4 \%$ & $9.6 \%$ & $41.0 \%$ \\
\hline & Moderate & $45.5 \%$ & $11.4 \%$ & $43.2 \%$ \\
\hline & Severe & $26.9 \%$ & $7.7 \%$ & $65.4 \%$ \\
\hline \multirow{3}{*}{$\begin{array}{l}\text { Extent to which their monthly income were affected } \\
\text { by the earthquake }\end{array}$} & Nothing & $71.8 \%$ & $2.8 \%$ & $25.4 \%$ \\
\hline & A little & $58.6 \%$ & $7.1 \%$ & $34.3 \%$ \\
\hline & Much & $38.9 \%$ & $16.7 \%$ & $44.4 \%$ \\
\hline
\end{tabular}

2009). However, this clinical presentation must be taken into account since the chance of PTSD recovery increase if there is no associated depression (Önder, Tural, Aker, Kiliç, \& Erdoğan, 2006).

Taking into consideration the characteristics of our sample, we should also be aware that the comorbidity between PTSD and depression has already been reported in young Haitians lacking social support after traumatic events (Fawzi et al., 2009). The need to address this issue goes beyond PTSD recovery, since this syndrome could have a cognitive impact, for example affecting memory (Eren-Koçak et al., 2009).

Regarding risk factors, consistent with the literature, we found a greater number of women in the High PTSD level, while there are more men in the Low level. This finding has also been reported by Brewin et al. (2000), and The American Psychological Association (2006) points out that the risk increases two-fold in women, depending on factors such as nature of the trauma.

On the other hand, age has proven to be controversial as a risk factor; in our study no differences were found, but some authors have reported that young age increases the risk (Brewin et al., 2000; Parvaresh \& Bahramnezhad, 2009), and others have reported that the risk increases with age (Zhang \& Ho, 2011). It is possible that in this case, age did not emerge as a risk factor due to the restriction of range in the sample, being all undergraduate students.

Among the students who were in Haiti, the proportion in both the High and Low PTSD level are similar, while among the students who were overseas, the majority of students are in the Low PTSD level. This is consistent with the findings of Armenian et al. (2000) and Chen et al. (2001) who indicate that closeness to the epicenter is a risk factor in the development of PTSD.

As to the relationship between family and personal history of mental illness and the development of PTSD symptoms our findings are in tune with those reported previously (Brewin et al., 2000; Campbell et al., 2007). Although no conclusive evidence has been found, the link between a history of mental illness and the development of PTSD is believed to be due to a physiological susceptibility (McMillen, North, Mosley, \& Smith, 2002).

The connection between the fear experienced during the earthquake and the symptoms of PTSD can be interpreted as a sign of high anxiety that could be, as explained by McNally (2008), considered as a risk factor. Those people could have been more affected during the event due to a preexisting tendency to suffer from an- 
xiety. It is also possible that an abnormal physiological response during the event might cause an irregular psychological state, as explained by Yehuda, McFarlane, \& Shalev (1998).

It is a well-known fact that being exposed to traumatic events during an earthquake is considered a risk factor (Zhang \& Ho, 2011). We decided to consider the sight of cadavers, body parts and wounded victims, not only from a direct experience but also through mass media, given that they may have either a positive or negative impact, depending on the central story (Lau et al., 2010).

In the case of seeing cadavers or body parts, these were not associated with the development of PTSD symptoms; however, a connection between seeing wounded people and the disorder has been observed. This might be due to it being easier to relate and empathize with people who are suffering (i.e., alive), whereas it might be more natural to objectify and dehumanize the dead.

Regarding the material losses, we noticed that the majority of people who had to change their living conditions after the earthquake were subject to various PTSD symptoms, which establishes this as a risk factor as posited by Parvaresh and Bahramnezhad's (2009) theory. Also, financial difficulties have already been identified in the literature as a stable risk factor for PTSD (Chou et al., 2007; Farhood \& Noureddine, 2003; Galea, Tracy, Norris, \& Coffey, 2008). In the student body, those who did not face financial difficulties were mainly in the lower levels of the disorder, while those who had to face important changes in their monthly income presented a higher prevalence of PTSD symptoms.

Considering these findings and keeping in mind that the university's student population is living on a tight budget, it is likely that this variable could make them more vulnerable to develop PTSD symptoms. Going further as to say that considering that the population of Haitian immigrants, as well as Haitians living in Haiti, are mostly poor (Brown et al., 2012), it is expected to observe a high percentage of PTSD symptoms not only in the student body but in the Haitian population in general. Factors such as these encourage more investigations about the subject, focusing in both working immigrants and general population in Haiti.

On the other hand, when it comes to death, students who suffered the loss of a family member fall into the category with the highest prevalence of PTSD symptoms. The quantity of deaths is a more important factor than the quality, as in which member died, since groups with fewer deaths reportedly presented fewer symptoms. This reflects the findings of different authors (e.g. Chou et al., 2007; Liu et al., 2010; Parvaresh \& Bahramnezhad, 2009) who report loss of family members as a risk factor in the development of PTSD. It has also been found that, especially with the works of Chou et al. (2007), the loss of friends and neighbors contributes to the development of the disorder, due to changes in the person's social life.

As to the effect of the satisfaction degree that the social support provides, results conflict: those who are not satisfied present fewer symptoms, whereas more than $40 \%$ of those who are satisfied display more symptoms. Although Brewin et al. (2000) identify the lack of social support as a risk factor, it is possible that those who fall into the category of high prevalence of PTSD value more the social support that they receive than those with fewer symptoms, probably because they did not have the need for it.

\section{Conclusion}

Some limitations are important to note. First, the population of Haitian students of PUCMM is not representative of the general Haitian population in terms of socio economic and demographic variables. Specifically, this sample of people had a higher socio economic status than most of the Haitian population and, as previously mentioned, the range of ages was restricted due to this being an undergraduate sample. Secondly, PTSD symptoms were measured by self-report and evaluations were not done by interview, but were filled out independently by the participants. Researchers supervised the administration of the measures and were available for questions. Moreover, a research culture has not been deeply rooted in the Dominican Republic, so recruiting participants presented a challenge. As much as the main motivation for the investigation was the possibility of affecting the creation of university policies based in their needs, the main reason for not participating was the reluctance to relive the traumatic experience.

Despite these limitations, we were able to identify important factors that may inform policymaking regarding the psychological management of undergraduate students following traumatic events. Some of those factors include the high incidence of PTSD symptoms in the sample (36\%), considering that other authors have reported rates between 5.7 (Liu et al., 2010) and 19.2\% (Önder et al., 2006) more than a year after an earthquake.

As mentioned before, there are multiple factors that would place the Haitian student population in the Dominican Republic at a higher risk level, such as being a minority in the country (Brewin et al., 2000), coming from 
and living in an underdeveloped country (Tural et al., 2004), which could explain why our results are higher than the ones observed by other authors.

Future studies should attempt to go beyond an undergraduate population and make efforts to recruit participants at the site of the earthquake.

\section{Acknowledgements}

Thank you to Daniel Jiménez for his valuable contributions to this paper and to the Pontificia Universidad Católica Madre y Maestra for hosting and funding this research.

\section{References}

American Psychiatric Association (2000). Diagnostic and Statistical Manual of Mental Disorders (4th ed.). Washington DC: American Psychiatric Association. http://dx.doi.org/10.1176/appi.books.9780890423349

American Psychological Association (2006). APA’s Response to International and National Disasters and Crises: Addressing Diverse Needs. American Psychologist, 61, 513-521. http://dx.doi.org/10.1037/0003-066X.61.5.513

Armenian, H. K., Morikawa, M., Melkonian, A. K., Hovanesian, A. P., Haroutunian, N., Saigh, P. A. et al. (2000). Loss as a Determinant of PTSD in a Cohort of Adult Survivors of the 1988 Earthquake in Armenia: Implications for Policy. Acta Psychiatrica Scandinavica, 102, 58-64. http://dx.doi.org/10.1034/j.1600-0447.2000.102001058.x

Ballenger, J. C., Davidson, J. R. T., Lecrubier, Y., Nutt, D. J., Marshall, R. D., Nemeroff, C. B. et al. (2004). Consensus Statement Update on Posttraumatic Stress Disorder from the International Consensus Group on Depressions and Anxiety. The Journal of Clinical Psychiatry, 65, 55-62.

Beck, A. T. (2006). Depression: Causes and treatment. Philadelphia: University of Pennsylvania Press.

Beck, A. T., \& Steer, R. A. (1993). Beck Anxiety Inventory Manual. San Antonio, TX: The Psychological Corporation Harcourt Brace \& Company.

Beck, A. T., Epstein, N., Brown, G., \& Steer, R. A. (1988). An Inventory for Measuring Clinical Anxiety: Psychometric Properties. Journal of Consulting and Clinical Psychology, 56, 893-897. http://dx.doi.org/10.1037/0022-006X.56.6.893

Beck, A. T., Ward, C. H., Mendelson, M., Mock, J., \& Erbaugh, J. (1961). An Inventory for Measuring Depression. Archives of General Psychiatry, 4, 561-571. http://dx.doi.org/10.1001/archpsyc.1961.01710120031004

Brewin, C. R., Andrews, B., \& Valentine, J. D. (2000). Meta-Analysis of Risk Factors for Posttraumatic Stress Disorder in Trauma-Exposed Adults. Journal of Consulting and Clinical Psychology, 68, 748-766. http://dx.doi.org/10.1037/0022-006X.68.5.748

Brown, C., Ripp, J., \& Kazura, J. (2012). Perspectives on Haiti Two Years after the Earthquake. The American Journal of Tropical Medicine and Hygiene, 86, 5-6. http://dx.doi.org/10.4269/ajtmh.2012.11-0684a

Campbell, D. G., Felker, B. L., Liu, C. F., Yano, E. M., Kirchner, J. E., Chan, D. et al. (2007). Prevalence of Depression-PTSD Comorbidity: Implications for Clinical Practice Guidelines and Primary Care-Based Interventions. Journal of General Internal Medicine, 22, 711-718. http://dx.doi.org/10.1007/s11606-006-0101-4

Chen, C. C., Yeh, T. L., Yang, Y. K., Chen, S. J., Lee, I. H., Fu, L. S. et al. (2001). Psychiatric Morbidity and Post-Traumatic Symptoms among Survivors in the Early Stage Following the 1999 Earthquake in Taiwan. Psychiatry Research, 105, 13-22. http://dx.doi.org/10.1016/S0165-1781(01)00334-1

Chou, F. H., Wu, H. C., Chou, P., Su, C. Y., Tsai, K. Y., Chao, S. S. et al. (2007). Epidemiologic Psychiatric Studies on Post-Disaster Impact among Chi-Chi Earthquake Survivors in Yu-Chi, Taiwan. Psychiatry and Clinical Neurosciences, 61, 370-378. http://dx.doi.org/10.1111/j.1440-1819.2007.01688.X

Choul, F. H., Chou, P., Lin, C., Su, T. T., Ou-Yang, W. C., Chien, I. C. et al. (2004). The Relationship between Quality of Life and Psychiatric Impairment for a Taiwanese Community Post-Earthquake. Quality of Life Research: An International Journal of Quality of Life Aspects of Treatment, Care and Rehabilitation, 13, 1089-1097. http://dx.doi.org/10.1023/B:QURE.0000031337.73269.64

Cohen, J. (1992). A Power Primer. Psychological Bulletin, 112, 155-159. http://dx.doi.org/10.1037/0033-2909.112.1.155

Cohen, S., Kamarck, T., \& Mermelstein, R. (1983). A Global Measure of Perceived Stress. Journal of Health and Social Behavior, 24, 385-396. http://dx.doi.org/10.2307/2136404

Eren-Koçak, E., Kiliç, C., Aydin, I., \& Hizli, F. G. (2009). Memory and Prefrontal Functions in Earthquake Survivors: Differences between Current and Past Post-Traumatic Stress Disorder Patients. Acta Psychiatrica Scandinavica, 119, 35-44. http://dx.doi.org/10.1111/j.1600-0447.2008.01281.x

Farhood, L. F., \& Noureddine, S. N. (2003). PTSD, Depression, and Health Status in Lebanese Civilians Exposed to a Church Explosion. International Journal of Psychiatry in Medicine, 33, 39-53. 
Fawzi, M. C., Betancourt, T. S., Marcelin, L., Klopner, M., Munir, K., Muriel, A. C. et al. (2009). Depression and Post-Traumatic Stress Disorder among Haitian Immigrant Students: Implications for Access to Mental Health Services and Educational Programming. BMC Public Health, 22, 482-492. http://dx.doi.org/10.1186/1471-2458-9-482

Foa, E. B., \& Tolin, D. F. (2000). Comparison of the PTSD Symptom Scale-Interview Version and the Clinician-Administered PTSD Scale. Journal of Traumatic Stress, 13, 181-191. http://dx.doi.org/10.1023/A:1007781909213

Galea, S., Tracy, M., Norris, F., \& Coffey, S. F. (2008). Financial and Social Circumstances and the Incidence and Course of PTSD in Mississippi during the First Two Years after Hurricane Katrina. Journal of Traumatic Stress, 21, 357-368. http://dx.doi.org/10.1002/jts.20355

Lau, J. T., Yu, X., Zhang, J., Mak, W. W., Choi, K. C., Lui, W. W. et al. (2010). Psychological Distress among Adolescents in Chengdu, Sichuan at 1 Month after the 2008 Sichuan Earthquake. Journal of Urban Health, 87, 504-523. http://dx.doi.org/10.1007/s11524-010-9447-3

Liu, Z. Y., Yang, Y. F., Ye, Y. L., Zeng, Z. Q., Xiang, Y. J., \& Yuan P. (2010). One-Year Follow-Up Study of Post-Traumatic Stress Disorder among Adolescents Following the Wenchuan Earthquake in China. BioScience Trends, 4, 96-102.

McMillen, C., North, C., Mosley, M., \& Smith, E. (2002). Untangling the Psychiatric Comorbidity of Posttraumatic Stress Disorder in a Sample of Flood Survivors. Comprehensive Psychiatry, 43, 478-485.

McMillen, J. C., North, C. S., \& Smith, E. M. (2000). What Parts of PTSD Are Normal: Intrusion, Avoidance, or Arousal? Data from the Northridge, California, Earthquake. Journal of Traumatic Stress, 13, 57-75. http://dx.doi.org/10.1023/A:1007768830246

McNally, R. J. (2008). Panic and Posttraumatic Stress Disorder: Implications for Culture, Risk, and Treatment. Cognitive Behaviour Therapy, 37, 131-134. http://dx.doi.org/10.1080/16506070801969120

Önder, E., Tural, U., Aker, T., Kiliç, C., \& Erdoğan, S. (2006). Prevalence of Psychiatric Disorders Three Years after the 1999 Earthquake in Turkey: Marmara Earthquake Survey (MES). Social Psychiatry and Psychiatric Epidemiology, 41, 868-874. http://dx.doi.org/10.1007/s00127-006-0107-6

Pan American Health Organization (2009). Health Situation Analysis and Trends Summary. http://www.haitiresiliencesystem.org/node/105

Parvaresh, N., \& Bahramnezhad, A. (2009). Post-Traumatic Stress Disorder in Bam-Survived Students Who Immigrated to Kerman, Four Months after the Earthquake. Archives of Iranian Medicine, 12, 244-249.

Pérez Benítez, C. I., Vicente, B., Zlotnick, C., Kohn, R., Johnson, J., Valdivia, S. et al. (2009). Estudio Epidemiológico de Sucesos Traumáticos, Trastorno de Estrés Post-traumático y otros Trastornos Psiquiátricos en una Muestra Representativa de Chile. Salud Mental, 31, 145-153.

Salcioglu, E., Basoglu, M., \& Livanou, M. (2007). Post-Traumatic Stress Disorder and Comorbid Depression among Survivors of the 1999 Earthquake in Turkey. Disasters, 31, 115-129. http://dx.doi.org/10.1111/j.1467-7717.2007.01000.x

Schnurr, P. P., Lunney, C. A., \& Sengupta, A. (2004). Risk Factors for the Development Versus Maintenance of Posttraumatic Stress Disorder. Journal of Traumatic Stress, 17, 85-95. http://dx.doi.org/10.1023/B:JOTS.0000022614.21794.f4

Silvestre, G., Anacréon, P., Théodore M., \& Silvestre, E. (2011). Prevalencia de trastorno de estrés post traumático en estudiantes haitianos un año tras el terremoto de enero 12 del 2010. Unpublished.

Tural, U., Coşkun, B., Önder, E., Çorapçioğlu, A., Yildiz, M., Kesepara, C. et al. (2004). Psychological Consequences of the 1999 Earthquake in Turkey. Journal of Traumatic Stress, 17, 451-459. http://dx.doi.org/10.1007/s10960-004-5793-9

Yehuda, R., McFarlane, A. C., \& Shalev, A. Y. (1998). Predicting the Development of Posttraumatic Stress Disorder from the Acute Response to a Traumatic Event. Biological Psychiatry, 44, 1305-1313. http://dx.doi.org/10.1016/S0006-3223(98)00276-5

Yuste, M. (2010). Un fuerte terremoto reduce a escombros la capital de Haití [A Strong Earthquake Reduces to Rubble Haiti’s Capital] El País. http://internacional.elpais.com/internacional/2010/01/12/actualidad/1263250811_850215.html

Zhang, Y., \& Ho, S. M. (2011). Risk Factors of Posttraumatic Stress Disorder among Survivors after the 512 Wenchuan Earthquake in China. PLoS ONE, 6, Article ID: e22371. http://dx.doi.org/10.1371/journal.pone.0022371 


\section{PTSD Symptom Scale (PSS)}

Adapted from: Foa, E. B., Riggs, D. S., Dancu, C. V., \& Rothbaum, B. O. (1993). Reliability and validity of a brief instrument for assessing posttraumatic stress disorder. Journal of Traumatic Stress, 6, 459-473

In the past two weeks (if $<2$ weeks since trauma, ask "Since the earthquake"). Probe all positive responses (e.g., "How often has this been happening?”)

\begin{tabular}{cccc}
\hline 0 & 1 & 2 & 3 \\
\hline not at & once per week & 2 to 4 times per & 5 or more times \\
all & or less/a little & week/somewhat & per week/very much \\
\hline
\end{tabular}

RE-EXPERIENCING (need one): [probe, then quantify]

1. Have you had recurrent or intrusive distressing thoughts or recollections about the earthquake?

2. Have you been having recurrent bad dreams or nightmares about the earthquake?

3. Have you had the experience of suddenly reliving the earthquake, flashbacks of it, acting or feeling as if it were re-occurring?

4. Have you been intensely EMOTIONALLY upset when reminded of the earthquake (includes anniversary reactions)?

5. Have you been having intense PHYSICAL reactions (e.g., sweaty, heart palpitations) when reminded of the earthquake?

AVOIDANCE (Need three): [probe, then qualify]

6. Have you persistently been making efforts to avoid thoughts or feelings associated with the earthquake?

7. Have you persistently been making efforts to avoid activities, situations, or places that remind you of the earthquake?

8. Are there any important aspects about the earthquake that you still cannot recall?

9. Have you markedly lost interest in free time activities since the earthquake?

10. Have you felt detached or cut off from others around you since the earthquake?

11. Have you felt that your ability to experience the whole range of emotions is impaired (e.g., unable to have loving feelings)?

12. Have you felt that any future plans or hopes have changed because of the assault (e.g., no career, marriage, children, or long life)?

INCREASED AROUSAL (need two): [probe then quantify]

13. Have you had persistent difficulty falling or staying asleep?

14. Have you been continuously irritable or have outbursts of anger?

15. Have you had persistent difficulty concentrating?

16. Are you overly alert (e.g., check to see who is around you, etc.) since the earthquake?

17. Have you been jumpier, more easily startled, since the earthquake? 